\title{
Fluxos de nutrientes em larvas de peixes: aplicação de técnicas com marcadores isotópicos e de modelação
}

\author{
Luís Conceição, Sofia Morais, Cláudia Aragão
}

\author{
CCMAR - Centro de Ciências do Mar, Universidade do Algarve,Campus de Gambelas, 8005-139 Faro, Portugal; e-mail: \\ lconcei@ualg.pt
}

RESUMO - Um modelo mecanístico que simule o crescimento durante a fase larvar de peixes deverá contribuir para um melhor conhecimento do processo de crescimento e respectivo metabolismo, com a intenção de elucidar as suas necessidades alimentares e desenvolver estratégias de alimentação adequadas. O modelo desenvolvido simula o crescimento e a evolução da composição bioquímica das larvas ao longo do tempo. O modelo depende essencialmente do consumo de alimento, sendo os nutrientes absorvidos usados para a produção de energia ou em processos de biossíntese e é baseado na estequiometria do metabolismo intermediário. Simulações utilizando este modelo sugerem que se deve prestar mais atenção a possíveis desequilíbrios no perfil de aminoácidos (AA) e a eventuais excessos de lípidos no alimento usado em cultivo larvares, de forma a optimizar o crescimento. Estudos usando marcadores isotópicos têm sido utilizados para estudar o metabolismo de AA e de lípidos em larvas de peixes e técnicas de modelação foram aplicadas de forma a permitir uma melhor compreensão dos resultados obtidos nestes estudos. A capacidade digestiva, bem como a utilização energética de diferentes AA, ácidos gordos e classes de lípidos, tem sido estudada utilizando a administração de nutrientes marcados (normalmente com ${ }^{14} \mathrm{C}$ ) directamente no tubo digestivo, através de um microcapilar. Este método permite a quantificação do nutriente marcado presente nas fezes, retido nos tecidos da larva e catabolizado. Um outro método, combinando a utilização de alimento vivo cujas proteínas estão marcadas com um isótopo estável com uma técnica espectroscópica/espectrométrica, permite a determinação do enriquecimento isotópico de AA individuais e pode ser usado para estimar o perfil ideal de AA indispensáveis na dieta. Em conjunto, estes dois tipos de técnicas de análise de fluxos de nutrientes têm permitido avanços importantes na compreensão da fisiologia da nutrição e crescimento de larvas de peixes.

Palavras-chave: estudos com marcadores, modelação mecanística, nutrição de larvas de peixes

\section{Nutrient fluxes in fish larvae: use of tracer studies and modelling techniques}

\footnotetext{
ABSTRACT - A mechanistic simulation model of fish larval growth should contribute to a better understanding of growth and metabolism of fish larvae. It should also assist in studying the nutritional requirements and developing feeding strategies. A model was developed that simulates growth and biochemical composition in time. The model is driven by feed intake, with absorbed nutrients being used for energy production and biosynthesis, based on the stoicheometry of intermediary metabolism. Model simulations suggest that imbalanced dietary amino acid (AA) profiles and dietary lipid excess may lead to suboptimal growth in fish larvae culture. Tracer studies have therefore been used to study AA and lipid metabolism in fish larvae. In addition, modeling techniques have been also used in these studies, to better interpret results obtained. Digestive capacity, as well as utilization of different AA, fatty acids and lipid classes, has been studied using radiolabeled nutrients (normally with ${ }^{14} \mathrm{C}$ ) using a tube-feeding technique. This method allows quantification of the tracer that is present in faeces, retained in tissues and catabolised. Another method, combining the use of live food in which proteins are labeled with a stable isotope and a spectroscopic/spectrometric technique that allows determination of the isotopic enrichment in individual AA, can be used to estimate ideal dietary indispensable AA profiles. Together these two types of techniques that allow the study of nutrient fluxes have lead to important advances in the understanding of fish larvae nutritional physiology and growth.
}

Key Words: fish larval nutrition, mechanistic modeling, tracer studies 


\section{Introdução}

Apesar de recentemente se terem vindo a observar progressos importantes na produção de juvenis de peixes marinhos, para muitas espécies esta produção continua a ser ainda insuficiente face ao requerido pela indústria aquícola. Em parte, isto deve-se a um conhecimento ainda limitado dos seus requisitos nutricionais (Watanabe \& Kiron, 1994; Shields, 2001). Em particular, é fundamental aprofundar o conhecimento da fisiologia nutricional das larvas de peixes, de forma a se proceder eficazmente a uma substituição dos organismos zooplanctónicos vivos usados na sua alimentação por microdietas inertes (Yúfera et al., 1996). As larvas de peixes marinhos, quando comparadas com as suas fases juvenis, têm uma capacidade reduzida de digerir e absorver nutrientes (e.g., Rust, 2002; Rønnestad \& Conceição, 2005), ainda que, paradoxalmente, tenham taxas de crescimento relativas muito superiores (Conceição et al., 1998). Este elevado potencial de crescimento implica, no entanto, que as larvas de peixes têm requisitos muito mais elevados em termos de aminoácidos (AA), ácidos gordos altamente insaturados (HUFA) e fosfolípidos (PL), entre outros nutrientes, ainda que os seus requisitos exactos sejam pouco conhecidos.

Quando se pretende estudar a digestão, absorção e utilização de nutrientes em larvas de peixes marinhos encontram-se vários problemas:

(1) as larvas da grande maioria de espécies de peixes marinhos, em particular as com interesse comercial, apresentam pequenas dimensões (cerca de 2-3 mm) à primeira alimentação;

(2) o tamanho da sua boca é também normalmente muito reduzido, sendo necessário que as partículas de alimento sejam muito pequenas (tipicamente 50-150 $\mu \mathrm{m}$ ), o que cria dificuldades técnicas para a produção de microdietas inertes;

(3) as larvas da maioria das espécies marinhas com interesse comercial não apresentam uma boa aceitação das microdietas inertes, o que resulta muitas vezes numa ingestão de alimento baixa e/ou muito variável em experiências de alimentação com larvas (Yúfera et al., 1996; Shields, 2001);
(4) é muito difícil manipular a composição nutricional do alimento vivo (rotíferos e artémia salina) normalmente utilizado (e.g., Kolkovski, 2001; Conceição et al., 2003a; Aragão et al., 2004a), com a excepção da fracção de lípidos neutros ou triacilgliceróis (TAG) e

(5) é muito difícil quantificar o consumo de alimento e a sua digestibilidade em larvas de peixes, o que torna a interpretação dos resultados de estudos nutricionais problemática.

Com vista a tentar solucionar parte destes problemas têm sido utilizadas técnicas de análise de fluxos de nutrientes, nomeadamente de modelação mecanística (e.g., Conceição et al., 1993, 1995, 1998; Conceição \& Rønnestad, 2004) e de marcadores isotópicos (e.g., Rønnestad et al., 2000 a,b, 2001 a,b; Conceição et al., 2001, 2002, 2003 b; Izquierdo et al., 2001; Hadas et al., 2003; Morais et al., 2004 a,b, 2005 a,b,c, 2006; Tonheim et al., 2004, 2005).

Este trabalho pretende fazer uma revisão das possíveis aplicações de técnicas de modelação mecanística e de marcadores isotópicos no estudo de fluxos de nutrientes em larvas de peixes, incluindo as suas vantagens e limitações.

\section{Modelação mecanística em larvas de peixes}

A modelação de sistemas é uma técnica com elevado potencial para melhor compreender os requisitos nutricionais das larvas de peixe. Um modelo mecanístico prediz o crescimento e composição bioquímica com base nos processos bioquímicos subjacentes (e.g., Machiels \& Henken, 1986; Gill et al., 1989; Baldwin \& Sainz, 1995). Este modelo é basicamente composto por um conjunto de equações que calculam a transferência de nutrientes entre as diferentes quantidades (pools) consideradas. Para além do seu poder de previsão, um modelo mecanístico pode ajudar a melhor compreender o processo em estudo (crescimento e metabolismo subjacente) e pode ainda identificar zonas obscuras do conhecimento, a serem estudadas no futuro. O desenvolvimento de um modelo mecanístico de crescimento é sempre um compromisso entre uma repre- 
sentação precisa dos processos envolvidos e a informação disponível para definir as equações que os representam e os seus parâmetros. A modelação pode ser vista como um processo contínuo de combinação entre a informação existente num modelo e o planeamento de novos estudos experimentais; quanto mais informação estiver disponível sobre o crescimento e a nutrição de larvas de peixe, mais se pode aperfeiçoar o modelo.

Um modelo mecanístico que simule o crescimento durante a fase larvar de peixes deverá contribuir para o conhecimento dos processos de crescimento e metabolismo associados, de forma a elucidar as necessidades alimentares larvares e desenvolver estratégias de alimentação adequadas. Conceição et al. $(1993,1998)$ desenvolveram modelos que simulam o crescimento e a evolução da composição bioquímica das larvas ao longo do tempo e que permitem ainda estimar as trocas de oxigénio, dióxido de carbono e amónia com o meio ambiente. O modelo depende essencialmente do consumo de alimento, sendo os nutrientes absorvidos usados para a produção de energia ou para os processos de biossíntese e é baseado na estequiometria do metabolismo intermediário. De acordo com o modelo, em períodos de elevado consumo de alimento e quando o perfil de AA na dieta é desequilibrado, as perdas elevadas de AA são inevitáveis e provocam um aumento na deposição de lípidos (Conceição et al., 1998). Simulações com o modelo também sugerem que um aumento no conteúdo de lípidos na dieta provoca um aumento no conteúdo lipídico das larvas, bem como um pequeno efeito de poupança de proteína (redução da proporção de AA utilizados para produção de energia e consequente aumento da sua deposição/retenção).

\section{Estudos com marcadores em larvas de peixes}

\section{Consumo de alimento}

A quantificação do consumo de alimento é essencial em qualquer estudo de nutrição, sem a qual não se pode compreender a eficiência de utilização dos nutrientes. Esta quantificação tornase ainda mais importante em estudos com larvas de peixes, pois o consumo de alimento está intimamente relacionado com o tempo de passagem do alimento através do tubo digestivo e logo com a eficiência de absorção dos nutrientes (Werner \& Blaxter, 1980; Boehlert \& Yoklavich, 1984; Morais et al., 2006). O uso de marcadores isotópicos para a quantificação do consumo de alimento é relativamente simples, podendo ser usados quer radioisótopos (p.ex., ${ }^{14} \mathrm{C},{ }^{35} \mathrm{~S}$ ou ${ }^{3} \mathrm{H}$ ) quer isótopos estáveis (p.ex., ${ }^{13} \mathrm{C}$ ou ${ }^{15} \mathrm{~N}$ ). No entanto, os radioisótopos são mais utilizados, dada a facilidade de quantificação, com elevada precisão, através de cintilação líquida. O inconveniente desta técnica é a limitação a estudos em volumes pequenos e confinados, por razões económicas e sobretudo de segurança. $\mathrm{O}$ uso de isótopos estáveis, ainda que seguro, é limitado pela menor sensibilidade e maior custo da sua quantificação. A quantificação do consumo pode ser efectuada usando radiomarcadores inseridos em nutrientes incorporados em microdietas (Kolkovski et al., 1993; Koven et al., 1998; Izquierdo et al., 2001; Hadas et al., 2003; Morais et al., 2006) ou em alimento vivo (Sorokin \& Panov, 1966; Govoni et al., 1982; Boehlert \& Yoklavich, 1984; Tandler \& Mason, 1984; Conceição et al., 1998; Morais et al., 2006). A marcação de alimento vivo com isótopos estáveis também já foi utilizada (Conceição et al., 2001). Mais recentemente, alguns trabalhos usaram as pequenas diferenças de abundância natural de isótopos estáveis (assinaturas isotópicas) para o estudo da selectividade de alimento por larvas de peixes (Jomori et al., 2005; Schlechtriem et al., 2005).

A maioria dos estudos existentes quantificou o consumo de alimento marcado com radioisótopos após uma única refeição. Este processo consiste em deixar as larvas alimentarem-se durante um período de tempo inferior ao tempo de trânsito no tubo digestivo, medindo-se depois a radioactividade na larva (Kolkovski et al., 1993; Koven et al., 1994, 1998; Conceição et al., 1998; Izquierdo et al., 2001). No entanto, estas medições são normalmente realizadas após um período de jejum e consequentemente representam o consumo máximo de alimento. Para se obter uma medida mais representativa da taxa de consumo de alimento, numa situação mais usual de alimentação em contínuo, devem fornecer-se refeições com dietas não marcadas antes e/ou depois do período de alimentação com a dieta marcada (Werner \& Blaxter, 1980; Morais et al., 2006).

๑ 2007 Sociedade Brasileira de Zootecnia 
Estudos que incluíram a quantificação do consumo de alimento usando este tipo de técnicas permitiram avanços importantes na nutrição de larvas de peixes marinhos. Kolkovski et al. (1993) demonstraram que uma das principais razões para o pouco sucesso da utilização de microdietas inertes em larvas de peixes marinhos se devia às baixas taxas de ingestão destas, sugerindo que a formulação e tecnologia de produção das microdietas teria de ser melhorada consideravelmente com vista a aumentar a sua atractividade. Koven et al. $(1994,1998)$ e Izquierdo et al. (2001), usando também microdietas marcadas com radioisótopos, verificaram um aumento do consumo de alimento em larvas de dourada (Sparus aurata) quando as microdietas continham níveis mais altos de fosfatidilcolina, sugerindo um efeito fagoestimulante para este lípido. Mais recentemente foi demonstrado que o nível de lípidos na dieta tem pouca influência na regulação do consumo de alimento em larvas de dourada, ao contrário do que parece acontecer em peixes juvenis e adultos e que a composição da dieta em ácidos gordos parece ser mais importante neste aspecto (Morais et al., 2006).

\section{Digestão e absorção de nutrientes}

A abordagem usualmente designada por hot chase é um método de análise de fluxos de nutrientes que consiste em seguir e quantificar a distribuição de um radioisótopo (p.ex., ${ }^{14} \mathrm{C},{ }^{35} \mathrm{~S}$, $\left.{ }^{3} \mathrm{H}\right)$ em diferentes compartimentos. Neste método, a larva é alimentada com presas vivas incorporando um radioisótopo durante um período de tempo inferior ao trânsito intestinal, ou, alternativamente, o nutriente marcado radioactivamente é administrado directamente no tubo digestivo da larva através de um microcapilar (processo conhecido como tube-feeding). Tipicamente, a distribuição do radioisótopo é analisada em diferentes compartimentos corporais (absorção ou retenção), na água de incubação das larvas (evacuação) e numa câmara metabólica (oxidação; ver abaixo). Deste modo, este método possibilita estudos de digestibilidade, assim como estimativas de taxas de absorção intestinal, catabolismo e retenção de diferentes nutrientes (ver abaixo). No caso mais simples, o conteúdo do radioisótopo é quantificado (por cintilação líquida) na larva e na água, uma vez terminado o processo digestivo (i.e., quando o tubo digestivo se encontra vazio), permitindo assim calcular a sua absorção (e.g., Rust et al., 1993; Rust, 1995). Este modelo simples, baseado em apenas dois compartimentos e com um único ponto de amostragem, pode adquirir diferentes graus de complexidade ao incluir compartimentos suplementares (p.ex., órgãos distintos) e/ou cinéticas de transferência entre compartimentos ou componentes específicos desses compartimentos (p.ex., AA livres ou proteínas; Rønnestad et al., 2000 a,b, 2001 a,b, 2003; Conceição et al., 2002; Rojas-García \& Rønnestad 2003 a,b; Aragão et al., 2004b; Morais et al., 2004 a,b, 2005 a,b,c; Tonheim et al., 2004, 2005). No entanto, o número de compartimentos passível de análise é grandemente limitado pelo exíguo tamanho das larvas, que dificulta a dissecção de órgãos distintos. Por exemplo, o tubo digestivo é normalmente facilmente dissecado mas nem sempre é possível isolar por completo órgãos distintos, como o fígado, que acabam por ser analisados conjuntamente, como parte de uma estrutura anatómica englobando vários órgãos. A principal limitação deste método para estudos de cinética de absorção intestinal é o facto da amostragem ter de ser feita após evacuação total do alimento radioisotópico. Isto devido à dificuldade em separar o tecido intestinal dos conteúdos luminais, o que poderia levar à inclusão de uma fracção variável ainda passível de ser evacuada. No entanto, desde que a amostragem seja feita em períodos superiores ao tempo de evacuação total da refeição radioisotópica, o método é válido e preciso para estimativas de digestibilidade ou de taxas de absorção de diferentes nutrientes ou alimentos.

De modo a estudar a digestibilidade e metabolismo proteico em larvas de peixes usando o método de tube-feeding é necessária uma proteína modelo que possa ser administrada numa solução fisiológica. Nos últimos anos têm sido desenvolvidos intensos esforços no sentido de sintetizar uma proteína modelo apropriada para este tipo de estudos, dado que as proteínas radioisotópicas actualmente disponíveis no mercado são marcadas através de ${ }^{14} \mathrm{C}$-metilação, o que altera as suas características químicas (Rojas-García \& Rønnestad, 2003 a,b; Rønnestad et al., 2003; Tonheim et al., 2004, 2005). Uma das abordagens testadas foi a adição de $\mathrm{H}^{14} \mathrm{CO}_{3}$ a 
cultivos de algas, mas o sucesso deste método foi limitado dado o baixo rendimento e pureza da proteína algal purificada, devido à contaminação por carbohidratos marcados (Tonheim et al., 2004). Um método mais eficaz foi a incorporação in vivo de AA marcados uniformemente em proteínas de serum de salmão (Tonheim et al., 2004, 2005). Hovde et al. (2005) desenvolveram um método mais sofisticado e com um elevado potencial de produção de proteínas especificamente sintetizadas para estudos precisos. Este consiste em inserir mRNA que se traduz em proteínas específicas em culturas bacterianas suplementadas com ${ }^{14} \mathrm{C}$-AA uniformemente marcados. No entanto, este método não é ainda correntemente aplicado dado o seu elevado custo e dificuldade técnica. Apesar destes constrangimentos, vários estudos realizados nos últimos anos utilizando uma ${ }^{14} \mathrm{C}$-proteína administrada através de tube-feeding e usando uma abordagem de hot chase permitiram confirmar teorias pré-existentes de que a ausência de um estômago funcional nas larvas de peixe afecta a sua capacidade de processamento das proteínas alimentares. Adicionalmente, foi demonstrado que esta limitação está associada essencialmente a uma baixa capacidade proteolítica e não absortiva do tubo digestivo, uma vez que AA livres, péptidos e hidrolisados proteicos são rápida e eficazmente absorvidos pelo sistema digestivo das larvas (e.g., Rust, 1995; Rojas-Garcia \& Rønnestad, 2003 a,b; Applebaum \& Rønnestad, 2004; Tonheim et al. 2004, 2005).

Uma outra metodologia utilizada para estudar a capacidade digestiva das larvas de peixe em relação à proteína alimentar é a marcação de náuplios ou metanáuplios de artémia salina através da adição de um $\left[\mathrm{U}-{ }^{14} \mathrm{C}\right]$ hidrolisado proteico ao meio de eclosão ou de enriquecimento (Morais et al. 2004a). Este método foi usado para examinar alterações ontogénicas na capacidade das larvas de linguado (Solea senegalensis) digerirem e metabolizarem os AA e proteínas que compõem a artémia, confirmando a hipótese que os enzimas intestinais e pancreáticos são eficientes mesmo nos estados larvares mais precoces (Morais et al. 2004b). Por outro lado, este método permite ainda comparar diferenças de digestibilidade da artémia salina entre diferentes espécies (Morais et al., 2004 a,b, 2005b). Uma vantagem deste método, relativamente à administração de proteínas radioisotópicas em solução (tube-feeding), é o de se tratar de uma situação mais representativa de um estado fisiológico normal. Sendo que uma solução líquida será muito provavelmente retida menos tempo no tubo digestivo da larva que uma refeição de artémia, o tempo de digestão e absorção será também inferior, levando potencialmente a subestimar a sua digestibilidade. A comparação de diversos estudos usando ambas abordagens parece confirmar esta hipótese (Govoni et al., 1982; Rust, 1995; Rønnestad et al., 2001a; Tonheim et al., 2004). A desvantagem do método é, no entanto, a impossibilidade de controlar a composição das proteínas que serão marcadas.

Para além dos estudos acima referidos usando proteínas, a abordagem de hot chase tem também sido utilizada para examinar se a forma em que um ácido gordo é administrado (livre ou esterificado a um TAG ou PL) afecta a sua digestão, absorção e metabolismo (Morais et al., 2005b). Estes estudos demonstraram que ácidos gordos livres são absorvidos mais eficientemente do que quando esterificados a TAG, indicando que o sistema digestivo da larva não é $100 \%$ eficaz para processar este tipo de lípidos. Por outro lado, quando o ácido gordo foi administrado esteri-ficado a um PL, a sua absorção por larvas de lin-guado foi bastante mais eficaz e comparável à do ácido gordo na sua forma livre (Morais et al., 2005b).

Uma segunda abordagem para estudar a digestão e absorção de nutrientes é conhecida como cold chase, devendo o seu nome ao facto de se utilizarem dietas não isotópicas (cold), após um período de alimentação (mais curto que o tempo de evacuação) com dietas idênticas mas marcadas com isótopos radioactivos ou estáveis. As larvas são amostradas após o período de alimentação com a dieta isotópica (hot) e depois periodicamente, após substituição da dieta pela idêntica não marcada, até à evacuação completa da dieta isotópica. Esta técnica foi originalmente desenvolvida por Sorokin \& Panov (1966) e foi utilizada em numerosos estudos, com pequenas adaptações (Govoni et al., 1982; Boehlert \& Yoklavich, 1984; Tandler \& Mason, 1984; Kolkovski et al., 1993; Conceição et al., 1998; Koven et al., 1998; Izquierdo et al., 2001; Hadas et al., 2003; Morais et al., 2006).

Um importante constrangimento desta metodologia consiste na dificuldade em remover

๑) 2007 Sociedade Brasileira de Zootecnia 
todos os vestígios da dieta isotópica da água de incubação, antes da sua substituição pela dieta não marcada, sem causar um stress significativo às larvas. Este procedimento poderá resultar em mortalidade ou alterações do comportamento alimentar, em maior ou menor grau, consoante a sensibilidade da espécie em estudo. Uma outra desvantagem, em comparação com o método de hot chase, onde o sistema experimental utilizado permite o estudo individual de larvas (Rønnestad et al., 2001a), é o facto de nas experiências de cold chase ser necessário analisar um conjunto de larvas (tipicamente 20-30 em triplicado; Kolkovski et al., 1993; Koven et al., 1998; Izquierdo et al., 2001; Hadas et al., 2003; Morais et al., 2006). Isto deve-se às particularidades desta metodologia e do sistema experimental utilizado, que implicam uma diluição bastante maior dos isótopos (por questões de custo), sendo no entanto de assinalar o aspecto positivo de uma maior segurança, no caso de exposição a radioisótopos. Outra desvantagem do método de cold chase é o facto do catabolismo não poder ser analisado com precisão, uma vez que neste sistema não existe uma armadilha química. Não obstante estas limitações, pelo facto de se poder estender o período de tempo durante o qual a ingestão e absorção dos alimentos é medida, a abordagem de cold chase tem algumas vantagens e diferentes aplicações relativamente à de hot chase, que têm sido exploradas em estudos recentes. De salientar, uma das aplicações mais comuns da abordagem cold chase tem sido o estudo da absorção de ácidos gordos pelo tecido intestinal (Kolkovski et al., 1993; Koven et al., 1994, 1998; Izquierdo et al., 2001; Morais et al., 2006).

\section{Catabolismo de nutrientes}

A metodologia utilizada em vários estudos para estimar as taxas de oxidação dos AA ou ácidos gordos recorre ao uso de compostos marcados com ${ }^{14} \mathrm{C}$. Este marcador pode ser dissolvido na água (Fauconneau et al., 1986), inserido em microdietas (Izquierdo et al., 2001) ou no alimento vivo (Morais et al., 2004a, 2005a), ou ainda administrado através de tube-feeding (Conceição et al., 2002; Aragão et al., 2004b; Morais et al., $2005 \mathrm{~b}, \mathrm{c})$. O fundamental em qualquer dos casos é a existência de uma armadilha química que retenha a fracção catabolizada $\left({ }^{14} \mathrm{CO}_{2}\right)$. Este

${ }^{\circledR} 2007$ Sociedade Brasileira de Zootecnia método foi inicialmente usado em juvenis de peixes (Walton et al., 1984 a,b; Fauconneau et al., 1992; Kim et al., 1992), mas foi também adaptado a larvas de esturjão (Fauconneau et al., 1986). Mais recentemente foi desenvolvido um método para larvas de peixes marinhos que permite a retenção do ${ }^{14} \mathrm{CO}_{2}$ resultante do metabolismo utilizando areja-mento e manipulando o $\mathrm{pH}$ da água de incubação (Rønnestad et al., 2001a).

Muitos dos estudos acima mencionados, relativos à digestão e absorção de proteínas e AA marcados com radioisótopos, também utilizaram uma armadilha química para retenção do ${ }^{14} \mathrm{CO}_{2}$ resultante do metabolismo, permitindo uma quantificação simultânea da utilização dos AA absorvidos como substratos para energia. Estes estudos demonstraram que, de uma forma geral, as larvas catabolizam uma grande parte dos AA absorvidos: cerca de $20 \%$ em larvas de roncadeirapinta (Leiostomus xanthurus) $10 \mathrm{~h}$ após ingestão (Govoni et al., 1982), 17\% em pós-larvas de alabote-do-Atlântico (Hippoglossus hippoglossus) 13h após tube-feeding (Rønnestad et al., 2001a), $39 \%$ em larvas de arenque (Clupea harengus) $24 \mathrm{~h}$ após a alimentação (Conceição et al., 2002) e 1129\% (Morais et al., 2004b, 2005a) em larvas de linguado (Solea senegalensis) $24 \mathrm{~h}$ após a alimentação. Noutros estudos em que, através de tube-feeding, se utilizou um único aminoácido marcado com ${ }^{14} \mathrm{C}$ foi demonstrado que as larvas são capazes de discriminar os diferentes AA (Conceição et al., 2003a): enquanto que os AA indispensáveis (IAA) são essencialmente retidos nos tecidos ( $>60 \%)$ e não catabolizados $(<25 \%)$, os AA dispensáveis apresentam um maior catabolismo (> 40\%) e uma menor retenção (< 57\%) (Rønnestad et al., 2001b; Conceição et al., 2002).

Estudos utilizando ácidos gordos livres marcados com ${ }^{14} \mathrm{C}$, fornecidos como componente de uma microdieta ou através de tube-feeding, demonstraram que diferentes ácidos gordos são metabolizados de forma diferente pelas larvas. Estes estudos evidenciaram que os ácidos gordos polinsaturados de cadeia longa, tais como os ácidos eicosapentaenóico (EPA) e docosahexaenóico (DHA), tendem a ser sobretudo retidos nos tecidos, possivelmente por serem preferencialmente reacilados em PL estruturais (e dado o seu papel essencial na nutrição larvar). Pelo contrário, o ácido oleico é sobretudo incorporado em TAG e 
preferencialmente catabolizado como fonte de energia (Izquierdo et al., 2001; Morais et al., 2005b).

\section{Retenção de aminoácidos}

Um método combinando a utilização de alimento vivo, cujas proteínas são marcadas com um isótopo estável, com uma técnica espectroscópica/espectrométrica permite a determinação do enriquecimento isotópico de AA individuais (Conceição et al., 2003b). Por sua vez, isto permite estimar o perfil ideal de IAA na dieta, ou seja, os requisitos qualitativos em IAA, de uma forma muito mais robusta que a simples utilização do perfil de IAA da carcaça dos peixes (Conceição et al., 2003a). Este método foi utilizado inicialmente usando rotíferos marcados com ${ }^{13} \mathrm{C}$ e combinando análises de AA (HPLC) com determinações do conteúdo em ${ }^{13} \mathrm{C}$ (nmol) por espectroscopia de ${ }^{13} \mathrm{C}$ NMR (Conceição et al., 2003b). Mais recentemente, este método foi utilizado usando rotíferos marcados com ${ }^{15} \mathrm{~N}$ e detecção por GC-C-IRMS (Saavedra et al., 2006). Em ambos os estudos os rotíferos foram marcados através da alimentação com microalgas enriquecidas no isótopo estável por um período de $48 \mathrm{~h}$. No entanto, a produção de microalgas enriquecidas em ${ }^{13} \mathrm{C}$ é mais complexa, pois exige a utilização de uma solução de $\mathrm{NaH}^{13} \mathrm{CO}_{3}$ (geradora de ${ }^{13} \mathrm{CO}_{2}$ ) num circuito de produção fechado, com recirculação do ar. A utilização de ${ }^{15} \mathrm{~N}$, em comparação com ${ }^{13} \mathrm{C}$-NMR, tem ainda as vantagens de permitir uma precisão bastante superior, pela utilização de GC-C-IRMS e de necessitar de enriquecimentos muito mais baixos de isótopo estável (i.e., mais económico).

Estas técnicas permitem determinar as biodisponibilidades relativas de cada AA, sendo esta calculada como a razão entre a actividade específica do isótopo estável de cada AA nas larvas e no alimento. Assim, foi possível demonstrar que há variações importantes de biodisponibilidades entre AA em larvas de peixes marinhos (Conceição et al., 2003b; Saavedra et al., 2006). A biodisponibilidade relativa é uma medida da eficiência de utilização de cada AA em relação à média de todos os outros AA e resulta do somatório da eficiência de absorção com a taxa de catabolismo de cada AA. A treonina, por exemplo, tem uma baixa biodisponibilidade relativa em larvas de dourada com 14 dias de idade, enquanto que a lisina, o aspartato e o glutamato têm elevadas biodisponibilidades relativas (Conceição et al., 2003b). Por outro lado, a isoleucina, leucina e valina têm biodisponibilidades relativas altas, enquanto que a alanina, glutamato e aspartato apresentam uma menor biodisponibilidade relativa em larvas de sargo bicudo (Diplodus puntazzo) com 8 dias de idade (Saavedra et al., 2006).

Os valores de biodisponibilidade relativa podem ser usados, em conjunto com o perfil de IAA das larvas, para calcular o perfil ideal de IAA nas dietas de larvas de peixes (Conceição et al., 2003 a,b). Este método pode ser usado como alternativa a estudos de dose-resposta, os quais são difíceis de realizar em larvas de peixes, para determinar os seus requisitos em AA. As vantagens deste método são a sua simplicidade em termos de zootecnia e o facto de permitir o estudo simultâneo da maioria dos AA.

\section{Integração de estudos com marcadores isotópicos e modelação}

As técnicas de modelação mecanística anteriormente referidas neste trabalho podem ser usadas para uma melhor interpretação dos resultados de estudos com marcadores isotópicos. Enquanto os estudos com marcadores isotópicos fazem medições pontuais durante o desenvolvimento (um ou mais pontos de amostragem), a modelação mecanística permite juntar a análise cinética da distribuição do marcador isotópico entre os diferentes compartimentos (pools) considerados no estudo.

De forma a melhor interpretar os resultados de trabalhos como o de Morais et al. (2004b), em que larvas de linguado foram alimentadas com uma refeição de artémia marcada com ${ }^{14} \mathrm{C}$-AA, Conceição \& Rønnestad (2004) desenvolveram um modelo mecanístico para o metabolismo de AA em larvas de peixes. O objectivo deste modelo é possibilitar uma melhor compreensão dos processos de digestão e absorção dos AA da dieta, bem como a sua posterior utilização metabólica. Este modelo é essencialmente controlado pelo consumo de AA da dieta, os quais, após absorção, se repartem entre a deposição de proteína e a produção de energia. O modelo permite a integração dos resultados obtidos após uma refeição com AA marcados, seguindo este

๑ 2007 Sociedade Brasileira de Zootecnia 
marcador nos pools de AA livres e proteína do tubo digestivo e da carcaça das larvas, ao longo do tempo. Simulações com o modelo sugerem que as taxas de síntese de proteínas e de catabolismo de AA aumentam rapidamente após a refeição, com um pico apenas $1 \mathrm{~h}$ depois e um retorno a níveis basais cerca de 3 h depois (Conceição \& Rønnestad, 2004). Isto significa que a digestão e processamento metabólico da proteína contida na artémia se efectua muito rapidamente e que, de forma a optimizar o potencial de crescimento de larvas de linguado, estas devem ser alimentadas com uma elevada frequência. Este modelo está ainda a ser aperfeiçoado e espera-se que contribua no futuro para uma melhor compreensão do metabolismo e da eficiência de utilização de AA pelas larvas de peixes.

\section{Conclusões}

Este trabalho demonstra que a aplicação de técnicas de análise de fluxos de nutrientes, nomeadamente usando marcadores isotópicos e modelação mecanística, tem permitido francos progressos no estudo da nutrição e crescimento de larvas de peixes. Mais especificamente, permitiram melhor compreender algumas das condicionantes da digestão, absorção e utilização de nutrientes em larvas de peixes marinhos. As técnicas de análise de fluxos de nutrientes, tais como as aqui descritas, constituem um novo conjunto de metodologias que terão certamente um papel importante em futuros estudos de nutrição larvar e a integração destes dois tipos de técnicas poderá acelerar o progresso nesta área do conhecimento.

Até ao momento ficou já bem demonstrado que se deve prestar mais atenção a possíveis desequilíbrios no perfil de AA e a eventuais excessos de lípidos, em particular TAG, no alimento usado em cultivo larvares, de forma a optimizar o crescimento. Mostrou-se ainda que as larvas de algumas espécies de peixes marinhos têm uma elevada capacidade digestiva, pelo menos no que se refere ao alimento vivo. No entanto, está agora bem estabelecido que a complexidade da fracção azotada da dieta é uma questão fulcral. As proteínas complexas (tais como as da farinha de peixe) são de difícil digestão durante os estádios iniciais de larvas de peixes marinhos. É também evidente que as larvas de peixes têm uma regulação estreita do seu metabolismo. Exemplificativo disto é a sua capacidade para discriminar diferentes AA e ácidos gordos conforme as suas necessidades, usando preferencialmente os nutrientes nãoessenciais para a produção de energia, poupando os essenciais/indispensáveis para a síntese de proteínas e outras moléculas, bem como para a sua integração em membranas e outras estruturas vitais.

\section{Literatura citada}

APPLEBAUM, S.; RØNNESTAD, I. Absorption, assimilation and catabolism of individual free amino acids by late larval Atlantic halibut (Hippoglossus hippoglossus). Aquaculture, v.230, p.313-322, 2004.

ARAGÃO, C.; CONCEIÇÃO, L.E.C.; DINIS, M.T. et al. Amino acid pools of rotifers and Artemia under different conditions: nutritional implications for fish larvae. Aquaculture, v.234, p.429-445, 2004a .

ARAGÃO, C.; CONCEIÇÃO, L.E.C.; MARTINS, D. et al. A balanced dietary amino acid profile improves amino acid retention in post-larval Senegalese sole (Solea senegalensis). Aquaculture, v.233, p.293-304, 2004 b.

BALDWIN, R.L.; SAINZ, R.D. Energy partitioning and modelling in animal nutrition. Ann. Rev. Nutr., v.15, p.191211, 1995.

BOEHLERT, G.W.; YOKLAVICH, M.M. Carbon assimilation as a function of ingestion rate in larval pacific herring, Clupea harengus pallasi Valenciennes. J. Exp. Mar. Biol. Ecol, v.79, p.251-262, 1984.

CONCEIÇÃO, L.; VERRETH, J.; SCHELTEMA, T. et al. A simulation model for the metabolism of yolk sac larvae of Clarias gariepinus. Aquacult. Fish. Manag., v.24, p.431443, 1993.

CONCEIÇÃO, L.; POLAT, A.; RØNNESTAD, I. et al. A first attempt to estimate protein turnover using a simulation model for amino acid metabolism in yolk sac larvae of Clarias gariepinus and Hippoglossus hippoglossus. ICES Mar. Sci. Symp, v. 201, p.80-86, 1995.

CONCEIÇÃO, L.E.C.; DERSJANT-LI, Y.; VERRETH, J.A.J. Cost of growth in larval and juvenile African catfish (Clarias gariepinus) in relation to growth rate, food intake and oxygen consumption. Aquaculture, v.161, p.95-106, 1998.

CONCEIÇÃO, L.E.C.; SKJERMO, J.; SKJÅK-BRÆK, G. et al. Effect of an immunostimulating alginate on protein turnover of turbot (Scophthalmus maximus L) larvae. Fish Physiol. Biochem, v.24, p.207-212, 2001.

CONCEIÇÃO, L.E.C.; RØNNESTAD, I.; TONHEIM, S.K. Metabolic budgets for lysine and glutamate in unfed herring (Clupea harengus) larvae. Aquaculture, v.206, p.305-312, 2002.

CONCEIÇÃO, L.E.C.; GRASDALEN, H.; RØNNESTAD, I. Amino acid requirements of fish larvae and post-larvae: new tools and recent findings. Aquaculture, v.227, p.221-232, $2003 \mathrm{a}$.

CONCEIÇÃO, L.E.C.; GRASDALEN, H.; DINIS, M.T. A new method to estimate the relative bioavailability of individual amino acids in fish larvae using 13C -NMR spectroscopy. Comp. Biochem. Physiol, v.134B, p.103-109, 2003 b.

CONCEIÇÃO, L.E.C.; RØNNESTAD, I. Utilisation of dietary amino acids in fish larvae: towards an explanatory model. In: AQUACULTURE EUROPE '04 BIOTECHNOLOGIES FOR QUALITY CONFERENCE, ADAMS, S.; OLAFSEN, J.A. (Eds.). EUROPEAN 
AQUACULTURE SOCIETY, SPECIAL PUBLICATION NO. 34, Oostende, Belgium, 2004. p.237-238.

FAUCONNEAU, B.; AGUIRRE, P.; DABROWSKI, K. et al. Rearing of sturgeon (Acipenser baeri Brandt) larvae. 2. Protein metabolism influence of fasting and diet quality. Aquaculture, v.51, p.117-131, 1986.

FAUCONNEAU, B.; BASSERES, A.; KAUSHIK, S.J. Oxidation of phenylalanine and threonine in response to dietary arginine supply in rainbow trout (Salmo gairdneri R.). Comp. Biochem. Physiol, v.101A, p.395-401, 1992.

GILL, M.; BEEVER, D.E.; FRANCE, J. Biochemical bases needed for the mathematical representation of whole animal metabolism. Nutr. Res. Rev., v.2, p.181-200, 1989.

GOVONI, J.J.; PETERS, D.S.; MERRINER, J.V. Carbon assimilation during larval development of the marine teleost Leiostomus xanthurus Lacépède. J. Exp. Mar. Biol. Ecol., v.64, p.287-299, 1982.

HADAS, E.; KOVEN, W.; SKLAN, D. et al. The effect of dietary phosphatidylcholine on the assimilation and distribution of ingested free oleic acid (18:1n-9) in gilthead seabream (Sparus aurata) larvae. Aquaculture, v.217, p.577588, 2003.

HOVDE, S.C.; VIDAL, M.C.; OPSTAD, I. et al. Design and synthesis of C-14-labelled proteins as tools for protein digestion studies in fish larvae. Aquacult. Nutr., v.11, n.6, p.395-401, 2005.

IZQUIERDO, M.S.; TANDLER, A.; SALHI, M. et al. Influence of dietary polar lipids quantity and quality on ingestion and assimilation of labeled fatty acids by larval gilthead seabream. Aquacult. Nutr., v.7, p.153-160, 2001.

JOMORI, R.K.; CARNEIRO, D.J.; DUCATTI, C. et al. Carbon and nitrogen stable isotopes (13C and $15 \mathrm{~N})$ as natural indicators of live food and in the Pacu Piractus mesopotamicus larval tissues. In: LARVI'05 - FISH \& SHELLFISH LARVICULTURE SYMPOSIUM, HENDRY, C.I.; VAN STAPPEN, G.; WILLE, M.; SORGELOOS, P. (Eds.). EUROPEAN AQUACULTURE SOCIETY, SPECIAL PUBLICATION NO. 36, Oostende, Belgium, 2005. p. 227-230.

KIM, K.; GRIMSHAW, T.W.; KAYES, T.B. et al. Effect of fasting or feeding diets containing different levels of protein or amino acids on the activities of the liver amino aciddegrading enzymes and amino acid oxidation in rainbow trout (Oncorhynchus mykiss). Aquaculture, v.107, p.89-105, 1992.

KOLKOVSKI, S.; TANDLER, A.; KISSIL, G.W.M. et al. The effect of dietary exogenous digestive enzymes on ingestion, assimilation, growth and survival of gilthead seabream (Sparus aurata, Sparidae, Linnaeus) larvae. Fish Physiol. Biochem., v.12, p.203-209, 1993.

KOLKOVSKI, S. Digestive enzymes in fish larvae and juveniles - implications and applications to formulated diets. Aquaculture, v.200, p.181-201, 2001

KOVEN, W.M.; HENDERSON, R.J.; SARGENT, J.R. Lipid digestion in turbot (Scopthalmus maximus). 2. Lipolysis invitro of C-14-labeled triacylglycerol, cholesterol ester and phosphatidylcholine by digesta from different segments of the digestive tract. Fish Physiol. Biochem., v.13, p.275-283, 1994.

KOVEN, W.M.; PARRA, G.; KOLKOVSKI, S. et al. The effect of dietary phosphatidylcholine and its constituent fatty acids on microdiet ingestion and fatty acid absorption rate in gilthead seabream, Sparus auratus, larvae. Aquacult. Nutr., v.4, p.39-45, 1998.

MACHIELS, M.A.M.; HENKEN, A.M. A dynamic simulation model for growth of the African Catfish, Clarias gariepinus (Burchell, 1822). I. Effect of feeding level on growth and energy metabolism. Aquaculture, v.56, p.29-52, 1986.

MORAIS, S.; CONCEIÇÃO, L.E.C.; DINIS, M.T. et al. A method for radiolabeling Artemia with applications in studies of food intake, digestibility, protein and amino acid metabolism in larval fish. Aquaculture, v.231, p.469-487, 2004a.

MORAIS, S.; LACUISSE, M.; CONCEIÇÃO, L.E.C. et al. Ontogeny of the digestive capacity of Senegalese sole (Solea senegalensis Kaup 1858), with respect to digestion, absorption and metabolism of amino acids from Artemia. Mar. Biol., v.145, p.243-250, 2004b.

MORAIS, S.; KOVEN, W.; RØNNESTAD, I. et al. Dietary protein/lipid ratio affects growth and amino acid and fatty acid absorption and metabolism in Senegalese sole (Solea senegalensis Kaup 1858) larvae. Aquaculture, v.246, p.347357, 2005a.

MORAIS, S.; KOVEN, W.; RØNNESTAD, I. et al. Dietary protein:lipid ratio and lipid nature affects fatty acid absorption and metabolism in a teleost larva. Br. J. Nutr., v.93, p.813820, 2005b.

MORAIS, S.; ROJAS-GARCIA, C.R.; CONCEIÇÃO, L.E.C. et al. Digestion and absorption of a pure triacylglycerol and a free fatty acid by Clupea harengus L. larvae. J. Fish Biol., v.67, p.223-238, 2005c.

MORAIS, S.; TORTEN, M.; NIXON, O. et al. Food intake and absorption are affected by dietary lipid level and lipid source in seabream (Sparus aurata L.) larvae. J. Exp. Mar. Biol. Ecol., v.331, p.51-63, 2006.

ROJAS-GARCÍA, C.R.; RØNNESTAD, I. Assimilation of dietary free amino acids, peptides and protein in post-larval Atlantic halibut (Hippoglossus hippoglossus). Mar. Biol., v.142, p.801-808, 2003a.

ROJAS-GARCÍA, C.R.; RØNNESTAD, I. Comment on the article "Assimilation of dietary free amino acids, peptides and protein in post-larval Atlantic halibut (Hippoglossus hippoglossus)". Mar. Biol., v.142, p.799, 2003b.

RØNNESTAD, I.; CONCEIÇÃO, L.E.C.; ARAGÃO, C. et al. Free amino acids are absorbed faster and assimilated more efficiently than protein in postlarval Senegal sole (Solea senegalensis). J. Nutr., v.130, p.2809-2812, 2000a.

RØNNESTAD, I.; PERÉZ DOMINGUEZ, R.; TANAKA, M. Ontogeny of digestive tract functionality in Japanese flounder, Paralichthys olivaceus studied by in vivo microinjection: $\mathrm{pH}$ and assimilation of free amino acids. Fish Physiol. Biochem., v.22, p. $225-235,2000 \mathrm{~b}$.

RØNNESTAD, I.; ROJAS-GARCÍA, C.R.; TONHEIM, S.K. et al. In vivo studies of digestion and nutrient assimilation in marine fish larvae. Aquaculture, v.201, p.161-175, 2001a.

RØNNESTAD, I.; CONCEIÇÃO, L.E.C.; ARAGÃO, C. et al. Assimilation and catabolism of dispensable and indispensable free amino acids in post-larval Senegal sole (Solea senegalensis). Comp. Biochem. Physiol., v.130C, p.461-466, $2001 \mathrm{~b}$.

RØNNESTAD, I.; TONHEIM, S.K.; FYHN, H.J. The supply of amino acids during early feeding stages of marine fish larvae: A review of recent findings. Aquaculture, v.227, p.147-164, 2003.

RØNNESTAD, I.; CONCEIÇÃO, L.E.C. Aspects of protein and amino acid digestion and utilization by marine fish larvae. In: STARCK, J.M.; WANG, T. (Eds.), Physiological and ecological adaptations to feeding in Vertebrates. Science Publishers, Enfield, New Hampshire, USA, 2005. p.389-416.

RUST, M.B.; HARDY, R.W.; STICKNEY, R.R. A new method for force-feeding larval fish. Aquaculture, v.116, p.341-352, 1993.

RUST, M.B. Quantitative aspects of nutrient assimilation in six species of fish larvae. University of Washington, USA, 1995. 150p. PhD thesis.

RUST, M.B. Nutritional Physiology. In: HALVER, J.E.; HARDY, R.W. (Eds.), Fish nutrition. Academic Press, Florida, USA, 2002. p. 368-446.

SAAVEDRA, M.; BELTRAN, M.; POUSÃO-FERREIRA, P. et al. Evaluation of bioavailability of individual amino acids in Diplodus puntazzo larvae: towards the ideal dietary amino

(0) 2007 Sociedade Brasileira de Zootecnia 
acid profile. Aquaculture, doi:10.1016/j.aquaculture.10.027, 2006.

SCHLECHTRIEM, C.; FOCKEN, U.; BECKER, K. Digestion and assimilation of the free-living nematode Panagrellus redivivus fed to first feeding coregonid larvae: Evidence from histological and isotopic studies. J. World Aquacult. Soc., v.36, p.24-31, 2005.

SHIELDS, R.J. Larviculture of marine finfish in Europe. Aquaculture, v.200, p.55-88, 2001.

SOROKIN, J.I.; PANOV, D.A. The use of ${ }^{14} \mathrm{C}$ for the quantitative study of the nutrition of fish larvae. Int. Rev. Ges. Hydrobiol, v.51, p.743-756, 1966.

TANDLER, A.; MASON, C. The use of ${ }^{14} \mathrm{C}$ labeled rotifers (Brachionus plicatilis) in the larvae of gilthead seabream (Sparus aurata): Measurements of the effect of rotifer concentration, the lighting regime and seabream larval age on their rate of rotifer ingestion. Eur. Maric. Soc., v.8, p.241259, 1984.

TONHEIM, S.K.; ESPE, M.; RAAE, A.J. et al. In vivo incorporation of $[\mathrm{U}]-{ }^{-14} \mathrm{C}$-amino acids: an alternative protein labelling procedure for use in examining larval digestive physiology. Aquaculture, v.235, p.553-567, 2004.

TONHEIM, S.K; ESPE, M.; HAMRE, K. et al. Pre-hydrolysis improves utilization of dietary protein in the larval teleost Atlantic halibut (Hippoglossus hippoglossus). J. Exp. Mar. Biol. Ecol., v.321, p.19-34, 2005.

WALTON, M.J.; COWEY, C.B.; ADRON, J.W. The effect of dietary lysine levels on growth and metabolism of rainbow trout (Salmo gairdneri). Br. J. Nutr., v.52, p.115-122,1984a.

WALTON, M.J.; COLOSO, R.M.; COWEY, C.B. The effects of dietary tryptophan levels on growth and metabolism of rainbow trout (Salmo gairdneri). Br. J. Nutr., v.51, p.279287, 1984b.

WATANABE, T.; KIRON, V. Prospects in larval fish dietetics. Aquaculture, v.124, p.223-251, 1994.

WERNER, R.G.; BLAXTER, J.H.S. Growth and survival of larval herring (Clupea harengus) in relation to prey density. Can. J. Fish. Aquat. Sci., v.37, p.1063-1069, 1980.

YÚFERA, M.; SARASQUETE, C.; FERNANDEZ-DÍAZ, C. Testing protein-walled microcapsules for the rearing of firstfeeding gilthead seabream, Sparus aurata L. Mar. Fresh. Res., v.47, p.211-216, 1996. 